\title{
Contractile Response to Sympathetic Innervation in Neonatal Ventricular Cardiomyocytes of the Spontaneously Hypertensive Rat
}

\author{
THOMAS R. LLOYD AND WILLIAM J. MARVIN, JR. \\ Steele Memorial Children's Research Center, University Heart Center, and Department of Pediatrics, University \\ of Arizona, Tucson, Arizona 85724 [T.R.L.] and Division of Pediatric Cardiology, Department of Pediatrics, \\ University of Florida Health Science Center, Jacksonville, FL 32209 [W.J.M.]
}

\begin{abstract}
Differences in cardiac development between spontaneously hypertensive rats (SHR) and their normotensive Wistar Kyoto (WKY) controls are observed before the onset of hypertension. To determine whether intrinsic differences in myocardium or autonomic neurons might be responsible for these observations, we studied primary cultures of isolated, never previously innervated ventricular cardiomyocytes from neonatal rats of both strains, and sympathetic innervation was produced by addition of neurons from thoracolumbar sympathetic ganglia. Both samestrain and cross-strain innervation were compared. Amplitude and frequency of contraction were measured from video images of spontaneously contracting cells by on-line video motion analysis. Sympathetic innervation improved contractile function by $61 \%$ in SHR cardiomyocytes $(p<$ 0.001 ), an effect qualitatively similar to that previously reported for WKY cardiomyocytes. Contractile function of SHR cardiomyocytes cultured without sympathetic explants was $25 \%$ less than that of WKY cells $(p<0.005)$, but the response of SHR cardiomyocytes to sympathetic innervation was twice as great as that of WKY myocytes $(p<0.01)$. Cross-strain innervation experiments showed that sympathetic neurons from both strains were equally effective; interstrain differences were confined to the cardiomyocytes. Interstrain differences in cardiomyocyte contractile function and contractile response to sympathetic innervation are present before the onset of hypertension, and may in part account for alterations in cardiac function observed in prehypertensive SHR. (Pediatr Res 30: 207210, 1991)
\end{abstract}

\section{Abbreviations}

SHR, spontaneously hypertensive rat WKY, Wistar Kyoto rat

Several investigators have documented cardiomyocyte hyperplasia and relative cardiomegaly in newborn SHR compared with the normotensive WKY strain (1-5). Despite SHR cardiomyocyte hyperplasia, cardiac contractility is lower in neonatal SHR than in WKY (3-5), implying that contractile function at the single-cell level is considerably reduced in the SHR. Because

Received January 4, 1991; accepted April 15, 1991.

Correspondence: Thomas R. Lloyd, M.D., Pediatric Cardiology, Arizona Health Sciences Center, Tucson, AZ 85724.

Supported by Grants HL35600 from the NHLBI, 82-1687 from the Arizona Disease Control Research Commission, and BRSG 2-S07RR05675-22 from the University of Arizona. T.R.L. was supported by National Research Service Award HL07413. blood pressure measurements have shown little (1-3) or no difference (4-6) between the strains in the first $3 \mathrm{wk}$ of life, these differences in cardiac mass and contractile function cannot be attributed to the hypertensive state.

Sympathetic innervation of the heart occurs in both strains during the first $21 \mathrm{~d}$ of life $(7,8)$, with coincident improvement in cardiac contractility. We have shown that in vitro sympathetic innervation causes substantial improvement in contractile function of cultured ventricular cardiomyocytes from newborn WKY (9). Sympathetic innervation also determines the vascular muscle phenotype of arterial segments transplanted between WKY and SHR through a trophic influence (10). Similarly, extracts of hypertrophic cardiac muscle from SHR can induce cardiac hypertrophy (11). Cardiac and vascular muscle from young SHR also exhibit increased sensitivity to adrenergic agonists (12-14). Based on these findings, we proposed that interstrain cellular differences in contractile function and in response to sympathetic innervation could produce a quantitatively different contractile effect in SHR that may in part account for the cardiac derangements observed in the absence of significant hypertension. This effect could be due to interstrain differences in the sympathetic neurons, the cardiomyocytes, or both.

We studied the contractile effects of sympathetic innervation on cultured newborn cardiomyocytes from the SHR and WKY strains using same-strain and cross-strain innervation. Comparing cardiomyocytes from both strains that were not allowed sympathetic innervation, we observed decreased contractile function in SHR. In contrast, contractile function of innervated cardiomyocytes was similar in both strains, indicating a larger increase in contractile function in SHR. Cross-strain innervation demonstrated that this increased response could be explained by interstrain differences in cardiomyocytes alone.

\section{MATERIALS AND METHODS}

Cell culture and interstrain comparison of noninnervated cardiomyocytes. Primary cultures of isolated ventricular myocytes were prepared from 1- to 3-d-old inbred rats of both SHR and WKY strains obtained from breeding colonies maintained at the University of lowa. At this age, sympathetic innervation of ventricular cardiomyocytes has not yet been achieved $(7,8)$. Separate instruments and glassware were used during the culture procedure to avoid cross contamination. The culture technique was as previously described $(9,15,16)$. The cardiac apex was used to avoid contamination with tissue from the atria or great vessels, and tissue from five to 10 pups from one or two litters was used for each primary culture. The tissue was minced, and cells were dissociated with trypsin. Cells were plated at a density of 200 to 300 viable (trypan blue exclusion) cells per $\mathrm{mm}^{2}$ of culture vessel surface to produce cultures consisting mostly of 207 
sisted of minimum essential medium-Earle's salts supplemented with $4 \mathrm{mM}$ L-glutamine, $15 \%$ horse serum, $20 \mu \mathrm{g} / \mathrm{mL}$ gentamicin, $20 \mathrm{mM} N$-2-hydroxyethylpiperazine- $N^{\prime}$-2-ethanesulfonic acid buffer, and $16 \mathrm{mM} \mathrm{NaHCO}_{3}$, and cultures were incubated at $37^{\circ} \mathrm{C}$ in a $5 \% \mathrm{CO}_{2}$ atmosphere. Culture medium was replaced with fresh medium after $2 \mathrm{~d}$, and contractile function was assessed, as described below, after an additional $2 \mathrm{~d}$ of incubation. Interstrain differences in contractile function of noninnervated cardiomyocytes were studied by comparing simultaneously prepared cultures from WKY and SHR pups.

Sympathetic innervation. Sympathetic innervation of SHR ventricular cardiomyocytes was produced as previously described $(9,16)$ by addition of minced thoracolumbar sympathetic ganglia from the SHR pups to myocyte cultures at the time of culture preparation. These neuronal explants consist predominantly of adrenergic neurons, as demonstrated by glyoxylic acid histofluorescence, which functionally innervate the myocytes within 72 $\mathrm{h}$, as indicated by myocyte responses to guanethidine and to electrical stimulation of axons (16). Again, culture medium was replaced after $2 \mathrm{~d}$, and contractile function measured after an additional $2 \mathrm{~d}$ of incubation. Response to sympathetic innervation was assessed by comparing innervated cardiomyocytes with simultaneously prepared cultures of cardiomyocytes without nerve explants.

Cross-strain innervation. To study cross-strain innervation, six types of cultures were simultaneously prepared: 1) WKY cardiomyocytes with WKY sympathetic explants (WKY samestrain innervation); 2) SHR cardiomyocytes with SHR sympathetic explants (SHR same-strain innervation); 3) WKY cardiomyocytes with SHR sympathetic explants (WKY cross-strain innervation); 4) SHR cardiomyocytes with WKY sympathetic explants (SHR cross-strain innervation); 5) WKY cardiomyocytes without sympathetic explants (WKY control); and 6) SHR cardiomyocytes without sympathetic explants (SHR control). These groups were compared to determine interstrain differences in cardiomyocytes and sympathetic neurons as outlined in Figure 1.

Contractile function. Contractile function was measured as previously described (9). Cardiomyocytes were studied in their culture medium, and temperature was held at $37^{\circ} \mathrm{C}$ by an air incubator. Cells were visualized by phase contrast microscopy (Leitz Diavert, Rockleigh, NJ) using a $32 \times$ objective lens, and the images of spontaneously contracting cardiomyocytes were converted to video using a closed circuit video camera. The motion of the cell membrane or other high contrast organelle was measured with a video motion detector (model no. 633; Colorado Video, Boulder, $\mathrm{CO}$ ) that produces a voltage signal proportional to the amplitude of contractile motion (17). This

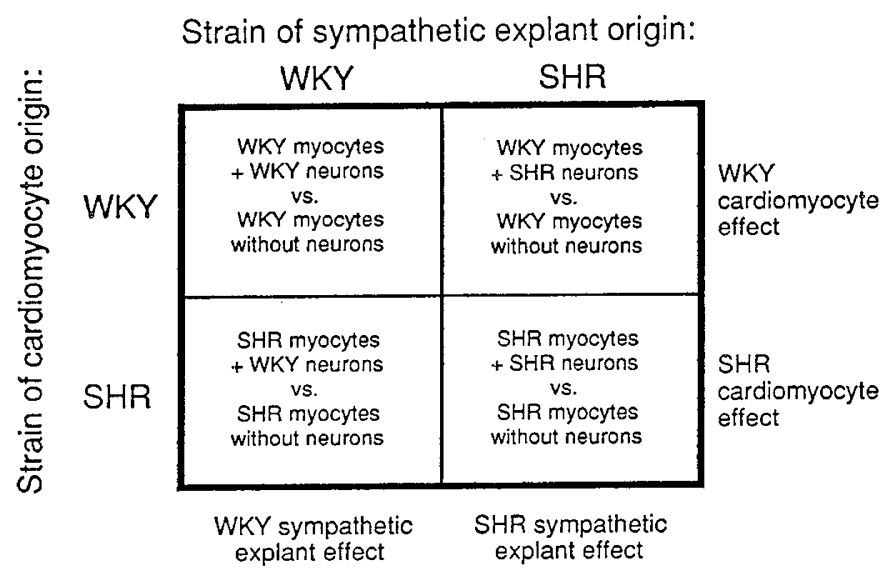

Fig. 1. Diagram of experimental design for determining interstrain differences in cardiomyocytes and sympathetic explants. Interstrain differences in cardiomyocytes are examined by comparing the rows, and interstrain differences in sympathetic explants are examined by comparing the columns. voltage signal was filtered $(7 \mathrm{~Hz}$ low pass) and calibrated to yield amplitude of contraction in $\mu \mathrm{m}$. We used this value as our index of contractile function. The amplitude signal was recorded at a paper speed of $25 \mathrm{~mm} / \mathrm{s}$, from which the rate of spontaneous contraction could be measured. Contraction amplitude was recorded from five randomly selected cardiomyocytes from each culture dish. This method has been shown to have excellent reproducibility and to be sensitive to changes in contractile function (9)

Statistical analysis. Our earlier study showed that contractile function varies substantially among primary cardiomyocyte cultures (9). All experiments were therefore repeated in at least four primary cultures, and analyses were conducted by nested analysis of variance, nesting for primary culture (when SHR cardiomyocytes given different treatments were compared) or for culture date (when SHR and WKY cardiomyocytes were compared). Nested one-factor analysis of variance was used for comparison of noninnervated WKY and SHR cardiomyocytes and for comparison of innervated and noninnervated SHR cardiomyocytes. Nested two-factor analysis of variance was used to compare the increase in contractile function (relative to control) of WKY and SHR cardiomyocytes subjected to same- and cross-strain innervation, the two factors being strain of cardiomyocyte origin and strain of neuronal origin (see Fig. 1). Data from a minimum of 40 cardiomyocytes was included in each analysis to provide an $80 \%$ likelihood of detecting a $25 \%$ change in contraction amplitude (two-tailed) at $p<0.05(9,18)$. Results are expressed as mean \pm SEM.

\section{RESULTS}

Interstrain comparison of noninnervated cardiomyocytes. Data from 85 SHR control cells were compared with 85 contemporaneously cultured WKY control cells. Amplitude of spontaneous contraction was $25 \%$ lower in SHR cardiomyocytes cultured without neurons than in similarly cultured WKY cells $(1.41 \pm$ 0.09 versus $1.89 \pm 0.11 \mu \mathrm{m}, p<0.005$ ). Frequency of spontaneous contraction was $86.2 \pm 4.4$ and $89.2 \pm 3.9 \mathrm{~min}^{-1}$ in SHR and WKY cells, respectively, which was not significantly different. Although amplitude of contraction varied substantially among different primary culture dates, values from SHR cardiomyocytes were consistently less than matched WKY values (Fig. 2).

Sympathetic innervation. The contractile effects of sympathetic innervation on SHR cardiomyocytes were studied in 45 innervated cells and in 45 control SHR cells from the same cultures. Amplitude of spontaneous contraction increased $61 \%$ with in-

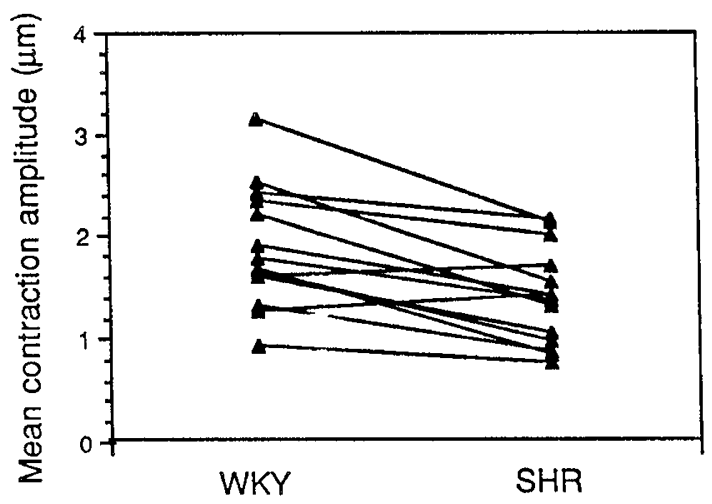

Fig. 2. Interstrain comparison: noninnervated cardiomyocytes. Lines connect mean values for amplitude of spontaneous contraction (in $\mu \mathrm{m}$ ) in 15 pairs of simultaneously prepared cultures of WKY and SHR cardiomyocytes without sympathetic neurons. Each point is the mean of data from five cardiomyocytes. In 13 paired cultures, contraction amplitude of WKY exceeded SHR; in only two pairs did SHR exceed WKY. Overall, amplitude of contraction in SHR cardiomyocytes was $25 \%$ lower than WKY $(p<0.005)$. 
nervation $(1.85 \pm 0.12$ versus $1.15 \pm 0.09 \mu \mathrm{m}, p<0.001)$ and spontaneous contraction frequency decreased $25 \%(63.2 \pm 4.5$ versus $84.6 \pm 5.6 \mathrm{~min}^{-1}, p<0.01$ ) with innervation.

Cross-strain innervation. Contractile function measurements were obtained from 25 cardiomyocytes in each of the six culture types described in Materials and Methods. Amplitude of contraction was $1.22 \pm 0.12 \mu \mathrm{m}$ in SHR control cells, $1.46 \pm 0.22$ $\mu \mathrm{m}$ in WKY control cells, $2.11 \pm 0.17 \mu \mathrm{m}$ in same-strain innervated SHR cells, $1.99 \pm 0.20 \mu \mathrm{m}$ in same-strain innervated WKY cells, $2.00 \pm 0.14 \mu \mathrm{m}$ in cross-strain innervated SHR cells, and $1.87 \pm 0.16 \mu \mathrm{m}$ in cross-strain innervated WKY cells. No significant differences in amplitude of contraction were found among the four groups of innervated cells, although each group had significantly higher values than the control cells $(p<0.05)$. However, when contractile response to innervation was compared by two-factor analysis of variance, the amplitude of contraction increased significantly more with innervation of SHR myocytes with neurons of either strain than with WKY myocytes ( 68 versus $32 \%, p<0.01$ ). No significant differences were present between the effects of WKY or SHR neurons in the experiment. These results are illustrated in Figure 3.

\section{DISCUSSION}

We observed significant differences in contractile function of isolated cardiomyocytes from newborn SHR and WKY. Contractile function of noninnervated SHR cardiomyocytes is reduced compared with WKY. Cardiomyocytes of both strains exhibit a positive contractile response to sympathetic innervation, but the contractile response to sympathetic innervation is greater in the SHR than in the WKY. We further demonstrated that this difference is due to the presence of interstrain differences in the cardiomyocytes.

Control cells. Amplitude of contraction was $25 \%$ lower in SHR control cells than in WKY, indicating reduced contractile function in noninnervated SHR cardiomyocytes. Potential mechanisms for this difference include biochemical differences in the contractile apparatus, electrochemical differences in membrane function, and ultrastructural differences in cell composition between the strains. Regardless of the explanation for the phenomenon, its occurrence may help explain the observation of cardiac hyperplasia in the newborn SHR that occurs before sympathetic innervation, when both strains have similar circulatory demands (1-5). If SHR cardiomyocytes function less effectively than WKY

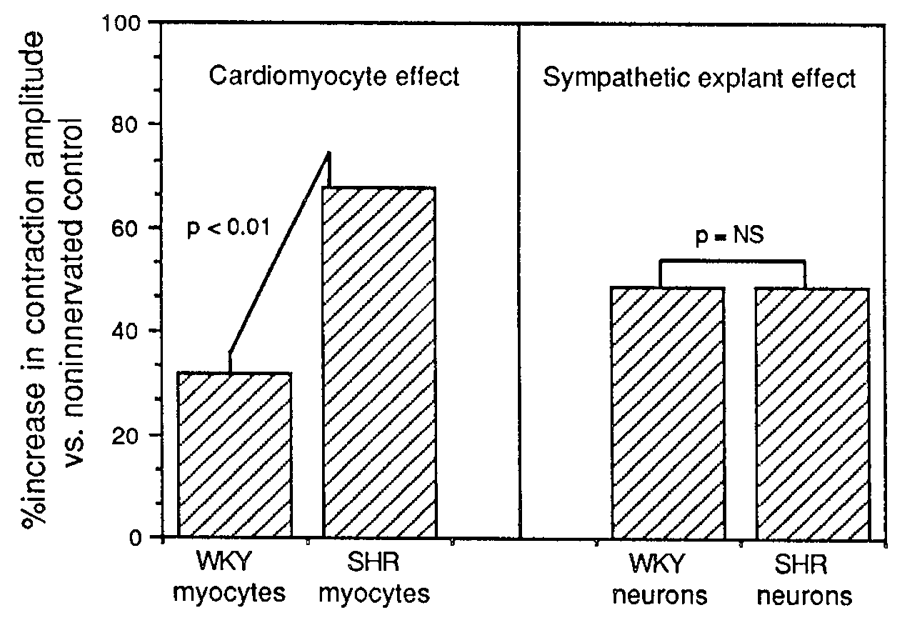

Fig. 3. Contractile effects of same-strain and cross-strain innervation on WKY and SHR cardiomyocytes is expressed as \% increase in contraction amplitude $v s$ noninnervated cardiomyocytes of the same strain. Data represent the row and column totals for interstrain comparison as described in Figure 1. Cardiomyocytes exhibited a significant $(p<0.01)$ interstrain difference regardless of the source of sympathetic explants (left panel), but there was no difference in the effectiveness of sympathetic neurons derived from the different strains (right panel). myocytes, more cardiomyocytes will be required to meet normal circulatory needs in the young SHR. Therefore, the relative cardiomegaly and cardiac hyperplasia observed at birth may be simply a compensatory response to normal circulatory demands.

Sympathetic innervation. The contractile and chronotropic responses to sympathetic innervation of SHR cardiomyocytes were qualitatively similar to those observed in the WKY (9). Although contractility varies with contraction frequency in a multiphasic fashion in the rat heart (19), contractility increases with contraction frequency over the range represented in this study. The contractile effect of sympathetic innervation is therefore unlikely to be due to the observed change in contraction frequency, because contractile function improves in the face of a negative chronotropic effect. The mechanisms by which sympathetic innervation improves contractile function remain unknown. We have previously reported (9) that the contractile effect of innervation in WKY is reproduced by conditioned medium from neuron-myocyte cocultures and is not inhibited by adrenoceptor blockade with phentolamine and propranolol; we have made similar observations in the SHR (data not shown). We have proposed a cotransmitter mechanism for this contractile effect (9), although such a mechanism must be considered speculative.

Cross-strain innervation. The cross-strain innervation experiments demonstrated that the contractile response to sympathetic innervation is significantly greater in SHR. The response to innervation was roughly twice as great in SHR cardiomyocytes as in WKY regardless of the strain from which the sympathetic neurons were taken, indicating that the interstrain difference in this system is confined to the cardiomyocytes. This finding is in contrast to the cross-strain innervation experiments of Abel and Hermsmeyer (10) using arterial segments transplanted to the anterior chamber of the eye. Arteries from young rats assumed the phenotype of the host animal after same- or cross-strain innervation, demonstrating that the interstrain difference was neuronal. Two crucial differences between the study of Abel and Hermsmeyer and our study are 1) the use of vascular smooth muscle instead of cardiac muscle and 2) the fact that our in vitro experiment exposed innervated cells only to the effects of sympathetic neurons from each strain, whereas the in vivo experiments of Abel and Hermsmeyer exposed the arterial segments to interstrain differences in CNS-mediated autonomic activity and to possible trophic factors present in the aqueous humor, as well as to sympathetic neurons.

Before sympathetic innervation occurs, the level of contractility is lower in SHR cardiomyocytes than in the WKY. After sympathetic innervation, SHR myocytes function as well as innervated WKY cells. Because in the intact animals the number of cardiomyocytes is greater in the SHR, the net effect of cardiac sympathetic innervation may be to encourage the "hyperdynamic circulation," which some have proposed is a precursor to hypertension in the young SHR (1-5).

Limitations. Many factors that affect cardiac function in neonatal animals are excluded from our tissue culture model, which allows unambiguous demonstration of effects due to sympathetic innervation. The absence of these same factors, however, limits direct extrapolation of our findings to the intact animal. The ratio of neurons to cardiomyocytes is supraphysiologic in our model, which limits quantitative estimation of the cardiac effect of sympathetic innervation in vivo (16). Although similar amounts of sympathetic explant material were added to all cocultures and no differences in the apparent density of axonal process development were noted between cultures, it is not possible to compare the extent of sympathetic innervation of the SHR and WKY myocytes studied in any meaningful way. It is therefore possible that some part of the increased contractile response to innervation exhibited by SHR cardiomyocytes might be due to some increased ability of these cells to foster sympathetic innervation. When directly comparing cells from different strains, we required that they be simultaneously cultured to 
reduce variability related to the solutions used or incubator conditions. Despite this care, some biologic variations may have existed between the SHR and WKY litters that were unrelated to strain differences, such as litter size or age of the dam. We have attempted to minimize any potential bias due to such influences by requiring that experiments be repeated on multiple primary cultures. Interpretation of the differences in response to innervation between WKY and SHR is complicated by the differences in contractility that exist before innervation. If there exists a developmental stage in which SHR and WKY myocytes have not developed differences, it must be antenatal, inasmuch as our cells from newborn rats already exhibited interstrain differences.

Conclusions. Differences in cardiomyocyte contractile function exist between SHR and WKY even in the neonatal period. Sympathetic innervation has a substantial positive contractile effect on cultured cardiomyocytes from both strains, but the effect is more pronounced in the SHR. These interstrain differences are found only in the cardiomyocyte; sympathetic neurons from the two strains appear to be interchangeable in this system. These interstrain differences develop before the onset of hypertension, and contribute to definition of the prehypertensive circulatory derangements in the SHR.

Acknowledgments. The authors thank Jane Rosenthal and Barbara Covey for technical assistance.

\section{REFERENCES}

1. Clubb FJ, Bell PD, Kriseman JD, Bishop SP 1987 Myocardial cell growth and blood pressure development in neonatal spontaneously hypertensive rats. Lab Invest 56:189-197

2. Walter SV, Hamet P 1968 Enhanced DNA synthesis in heart and kidney of newborn spontaneously hypertensive rats. Hypertension 8:520-525

3. Yamori Y, Mori C, Nishio T, Ooshima A, Horie R, Ohtaka M, Soeda T, Saito M, Abe K, Nara Y, Nakao Y, Kihara M 1979 Cardiac hypertrophy in early hypertension. Am J Cardiol 44:964-969

4. Cutilletta AF, Benjamin M. Culpepper WL, Oparil S 1968 Myocardial hyper- trophy and ventricular performance in the absence of hypertension in spontaneously hypertensive rats. J Mol Cell Cardiol 10:689-703

5. Sen S, Bumpus FM 1979 Collagen synthesis in development and reversal of cardiac hypertrophy in spontaneously hypertensive rats. Am J Cardiol 44:954-958

6. Lais L, Rios LL, Boutelle S, Dibona GF, Brody MJ 1977 Arterial pressure development in neonatal and young spontaneously hypertensive rats. Blood Vessels 14:277-284

7. Pappano A 1977 Ontogenetic development of autonomic neuroeffector transmission and transmitter reactivity in embryonic and fetal hearts. Pharmacol Rev 29:3-33

8. Lais LT, Brody MJ, Bhatnagar RK, Roskoski R 1976 Evidence that hypertension appears in SHR in the absence of altered sympathetic nervous system activity or development. In: Okamoto K, Bumpus F (eds) Spontaneous Hypertension: Its Pathogenesis and Complications. U.S. Government Printing Office, Washington, DC, pp 181-190

9. Lloyd TR, Marvin WJ 1990 Sympathetic innervation improves the contractile performance of neonatal cardiac ventricular myocytes in culture. J Mol Cell Cardiol 22:333-342

10. Abel PW, Hermsmeyer K 1981 Sympathetic cross-innervation of SHR and genetic controls suggest a trophic influence on vascular muscle membranes. Circ Res 49:1311-1318

11. Sen S, Petscher C, Ratliff N 1987 A factor that initiates myocardial hypertrophy in hypertension. Hypertension 9:261-267

12. Blumenthal SJ, McConnaughey MM, lams SG 1982 Myocardial adrenergic receptors and adenylate cyclase in the developing spontaneously hypertensive rat. Clin Exp Hypertens (A) 4:883-901

13. Hermsmeyer K 1976 Cellular basis for increased sensitivity of vascular smooth muscle in spontaneously hypertensive rats. Circ Res (suppl 2) 38:53-57

14. Lais LT, Brody MJ 1978 Vasoconstrictor hyperresponsiveness: an early pathogenic mechanism in the spontaneously hypertensive rat. Eur $\mathbf{J}$ Pharmacol 47:177-189

15. Marvin WJ, Robinson RB, Hermsmeyer K 1979 Functional and morphological properties of cultured cardiac and vascular muscle cells. Circ Res 45:528propert

16. Marvin WJ, Atkins DL, Chittick VL, Lund DD, Hermsmeyer K 1984 In vitro adrenergic and cholinergic innervation of the developing rat myocyte. Circ Res 55:49-58

17. Biedert S, Barry WH, Smith TW 1979 Inotropic effects and changes in sodium and calcium contents associated with inhibition of monovalent cation active transport by ouabain in cultured myocardial cells. J Gen Physiol 74:479494

18. Lachin JM 1981 Introduction to sample size determination and power analysis for clinical trials. Controlled Clin Trials 2:93-113

19. Meijler FL 1983 Staircase, rest contractions, and potentiation in the isolated rat heart. Am J Physiol 202:636-640 